\title{
Modified Signed Log-Likelihood Ratio Test for Comparing the Correlation Coefficients of Two Independent Bivariate Normal Distributions
}

\author{
Mohammad Reza Kazemi ${ }^{\dagger}$ and Ali Akbar Jafari ${ }^{\ddagger}, *$ \\ ${ }^{\dagger}$ Fasa University \\ ¥ Yazd University
}

Received: 11/30/2014 Approved: 5/30/2016

\begin{abstract}
In this paper, we use the method of modified signed log-likelihood ratio test for the problem of testing the equality of correlation coefficients in two independent bivariate normal distributions. We compare this method with two other approaches, Fisher's Z-transform and generalized test variable, using a Monte Carlo simulation. It indicates that the proposed method is better than the other approaches, in terms of the actual sizes and powers especially when the sample sizes are unequal. We illustrate performance of the proposed approach, using a real data set.
\end{abstract}

Keywords. Bivariate normal distribution; actual size; correlation coefficient; maximum likelihood estimator; power.

MSC 2010: 62F03, 62H10, 62E20

${ }^{*}$ Corresponding author 


\section{Introduction}

The linear association between two normal variables is usually measured by correlation coefficient. Statistical inferences for this parameter are divided to a single bivariate sample and several bivariate samples problems. In the case of a single sample, Fisher (1915) for the first time and then Hotelling (1953) provided the exact density function of product moment correlation coefficient. In testing and constructing the confidence interval for the correlation coefficient, Fisher (1921) introduced the well-known Fisher Z-transform, Sun and Wong (2007) proposed a likelihood-based higher-order asymptotic method, and Krishnamoorthy and Xia (2007) proposed a generalized pivotal approach. Kazemi and Jafari (2015) compared some confidence intervals for the correlation coefficient.

The problem of equality of two correlations arises practically, for example in comparing the correlations between the laterality of blood flow in each brain region and verbal memory score across gender (see Bilker et al., 2004). For inference about this problem, Zar (1999) used the Fisher Z-transform to test that whether all samples came from populations having common correlation coefficient, and Olkin and Finn (1995) obtained an asymptotic distribution of the difference between two sample correlation coefficients. Krishnamoorthy and Xia (2007) proposed a generalized test variable and studied the performances of this test, Fisher Z-transform test and Olkin and Finn's method. They concluded that Olkin and Finn's method is satisfactory for large sample sizes, and Fisher Z-transform test is conservative (i.e. its actual size is very smaller than the nominal level) when the samples are small. In addition, the actual size of generalized test variable is close to the nominal level for moderate samples.

The aim of this paper is to develop a modified signed log-likelihood ratio (MSLR) method for testing the equality of two correlation coefficients in two independent bivariate normal distributions. We used the test statistic proposed by DiCiccio et al. (2001) which has a simple form and then applied the traditional signed log-likelihood ratio (SLR) test in its form. We propose a parametric bootstrap method to approximate the distribution of SLR statistic and then use it to compute the MSLR statistic. Our simulation results show that MSLR test always are satisfactory regardless of the sample sizes and values of the common correlation coefficient.

This paper is organized as follows: Some preliminaries are given in Section 2. The MSLR is explained for testing the equality of two correlation coeffi- 
cients in Section 3. In Section 4, a simulation study is performed to evaluate and compare the actual sizes and powers of MSLR, Fisher's Z-transform and generalized variable approaches. Also, the approaches are illustrated using a real example.

\section{Preliminaries}

Let $\left(X_{i j}, Y_{i j}\right), i=1,2$ and $j=1,2, \ldots, n_{i}$ be a random sample from the bivariate normal distribution with mean vector $\boldsymbol{\mu}_{i}=\left(\mu_{1 i}, \mu_{2 i}\right)^{\prime}$ and variance covariance matrix

$$
\Sigma_{i}=\left[\begin{array}{cc}
\sigma_{1 i}^{2} & \rho_{i} \sigma_{1 i} \sigma_{2 i} \\
\rho_{i} \sigma_{1 i} \sigma_{2 i} & \sigma_{2 i}^{2}
\end{array}\right], \quad i=1,2 .
$$

Our goal is to test the hypothesis

$$
H_{0}: \rho_{1}=\rho_{2}=\rho \quad \text { vs. } \quad H_{1}: \rho_{1} \neq \rho_{2},
$$

where $\rho$ is the common correlation coefficient. We use the method of SLR for this problem. To apply this method, we need to find the full and constrained maximum likelihood estimators (MLE) of the unknown model parameters. Considering $\boldsymbol{\theta}=\left(\boldsymbol{\theta}_{1}, \boldsymbol{\theta}_{2}\right)$, where $\boldsymbol{\theta}_{i}=\left(\mu_{1 i}, \mu_{2 i}, \sigma_{1 i}, \sigma_{2 i}, \rho_{i}\right)$, it can be shown that the $\log$-likelihood function can be written as

$$
\ell(\boldsymbol{\theta})=c+\ell_{1}\left(\boldsymbol{\theta}_{1}\right)+\ell_{2}\left(\boldsymbol{\theta}_{2}\right),
$$

where

$$
\begin{aligned}
\ell_{i}\left(\boldsymbol{\theta}_{i}\right)= & -n_{i} \log \left(\sigma_{1 i}\right)-n_{i} \log \left(\sigma_{2 i}\right)-\frac{n_{i}}{2} \log \left(1-\rho_{i}^{2}\right) \\
& -\frac{n_{i} \mu_{1 i}^{2}}{2\left(1-\rho_{i}^{2}\right) \sigma_{1 i}^{2}}-\frac{n_{i} \mu_{2 i}^{2}}{2\left(1-\rho_{i}^{2}\right) \sigma_{2 i}^{2}} \\
& +\frac{n_{i} \rho_{i} \mu_{1 i} \mu_{2 i}}{2\left(1-\rho_{i}^{2}\right) \sigma_{1 i} \sigma_{2 i}}-\frac{\rho_{i} \sum_{k=1}^{n_{i}} x_{i j}^{2}}{2\left(1-\rho_{i}^{2}\right) \sigma_{1 i}^{2}} \\
& -\frac{\rho_{i} \sum_{j=1}^{n_{i}} y_{i j}^{2}}{2\left(1-\rho_{i}^{2}\right) \sigma_{2 i}^{2}}+\frac{\left(\mu_{1 i} \sigma_{2 i}-\mu_{2 i} \sigma_{1 i}\right) \sum_{k=1}^{n_{i}} x_{i j}}{\left(1-\rho_{i}^{2}\right) \sigma_{1 i}^{2} \sigma_{2 i}}
\end{aligned}
$$




$$
+\frac{\left(\mu_{2 i} \sigma_{1 i}-\mu_{1 i} \sigma_{2 i}\right) \sum_{k=1}^{n_{i}} y_{i j}}{\left(1-\rho_{i}^{2}\right) \sigma_{2 i}^{2} \sigma_{1 i}}+\frac{\rho_{i} \sigma_{1 i} \sum_{j=1}^{n_{i}} x_{i j} y_{i j}}{\left(1-\rho_{i}^{2}\right) \sigma_{1 i} \sigma_{2 i}} .
$$

It is known that, under the full model (without any constraint), the MLE's of parameters $\boldsymbol{\theta}_{i}$ are $\hat{\boldsymbol{\theta}}_{i}=\left(\bar{X}_{i}, \bar{Y}_{i}, S_{1 i}, S_{2 i}, R_{i}\right), i=1,2$, where

$$
\begin{gathered}
\bar{X}_{i}=\frac{1}{n_{i}} \sum_{j=1}^{n_{i}} X_{i j}, \quad \bar{Y}_{i}=\frac{1}{n_{i}} \sum_{j=1}^{n_{i}} Y_{i j}, \quad R_{i}=\frac{S_{12(i)}}{\sqrt{S_{1 i}^{2} S_{2 i}^{2}}} \\
S_{1 i}^{2}=\frac{1}{n_{i}} \sum_{j=1}^{n_{i}}\left(X_{i j}-\bar{X}_{i}\right)^{2}, \quad S_{2 i}^{2}=\frac{1}{n_{i}} \sum_{j=1}^{n_{i}}\left(Y_{i j}-\bar{Y}_{i}\right)^{2},
\end{gathered}
$$

and $S_{12(i)}=\sum_{j=1}^{n_{i}}\left(X_{i j}-\bar{X}_{i}\right)\left(Y_{i j}-\bar{Y}_{i}\right)$.

For the constrained model i.e. under the hypothesis in (1), Pearson (1933) showed that the MLE of the common correlation coefficient, $\tilde{\rho}$, is obtained by solving the following equation:

$$
\frac{n_{1}\left(r_{1}-\tilde{\rho}\right)}{\left(1-\tilde{\rho} r_{1}\right)}+\frac{n_{2}\left(r_{2}-\tilde{\rho}\right)}{\left(1-\tilde{\rho} r_{2}\right)}=0
$$

where $r_{i}$ is the observed value of $R_{i}$ (For more details, refer to Pearson, 1933; Donner and Rosner, 1980). Also, the constrained MLE's of parameters $\mu_{1 i}$, $\mu_{2 i}, \sigma_{1 i}^{2}$ and $\sigma_{2 i}^{2}$ are

$$
\tilde{\mu}_{1 i}=\bar{x}_{i}, \quad \tilde{\mu}_{2 i}=\bar{y}_{i}, \quad \tilde{\sigma}_{1 i}^{2}=\frac{s_{1 i}^{2}\left(1-\tilde{\rho} r_{i}\right)}{1-\tilde{\rho}^{2}}, \quad \tilde{\sigma}_{2 i}^{2}=\frac{s_{2 i}^{2}\left(1-\tilde{\rho} r_{i}\right)}{1-\tilde{\rho}^{2}}
$$

where $\bar{x}_{i}, \bar{y}_{i}, s_{1 i}^{2}$ and $s_{2 i}^{2}$ are the observed value of $\bar{X}_{i}, \bar{Y}_{i}, S_{1 i}^{2}$ and $S_{2 i}^{2}$. In this case, the MLE of parameter $\boldsymbol{\theta}_{i}$ is $\tilde{\boldsymbol{\theta}}_{i}=\left(\tilde{\mu}_{1 i}, \tilde{\mu}_{2 i}, \tilde{\sigma}_{1 i}, \tilde{\sigma}_{2 i}, \tilde{\rho}\right)$.

Donner and Rosner (1980) defined

$$
R_{F}=\frac{\exp (2 \bar{Z})-1}{\exp (2 \bar{Z})+1}=\tanh (\bar{Z})
$$

where $\bar{Z}=\frac{\left(n_{1}-3\right) Z_{1}+\left(n_{2}-3\right) Z_{2}}{n_{1}+n_{2}-6}$ and $Z_{i}=\frac{1}{2} \log \left(\frac{1+R_{i}}{1-R_{i}}\right)=\tanh ^{-1}\left(R_{i}\right), i=1,2$. They showed that the estimators $\tilde{\rho}$ and $R_{F}$ are close when the samples sizes are equal, i.e. $n_{1}=n_{2}$. Simulation studies (not reported here) show that the results for MSLR method based on the estimators $\tilde{\rho}$ and $R_{F}$ are close to 
each other. But the estimator $R_{F}$ decrease the execution time. Therefore, $R_{F}$ can be used instead of $\tilde{\rho}$ to estimate the common correlation coefficient $\rho$.

The following lemma helps us to generate the sample correlation coefficient from a random sample of a bivariate normal distribution. It is notable that this formula is different from formula (16) of Krishnamoorthy and Xia (2007) and also it cannot be used as the generalized pivotal quantity.

Lemma 1. Let $R_{i}$ be the sample correlation coefficient from a bivariate normal distribution with mean vector $\boldsymbol{\mu}_{i}$ and variance-covariance matrix $\Sigma_{i}$. Then

$$
R_{i} \stackrel{d}{=} \frac{\rho_{i}^{*} V_{i}+N_{i}}{\sqrt{\left(\rho_{i}^{*} V_{i}+N_{i}\right)^{2}+W_{i}^{2}}},
$$

where $\rho_{i}^{*}=\frac{\rho_{i}}{\sqrt{1-\rho_{i}^{2}}}$, and $V_{i}^{2}, W_{i}^{2}$, and $N_{i}$ are independent random variables with $\chi_{(n-1)}^{2}, \chi_{(n-2)}^{2}$ and $N(0,1)$ distributions, respectively.

Proof. Let $S_{i}=\left[\begin{array}{cc}S_{1 i}^{2} & S_{12(i)} \\ S_{12(i)} & S_{2 i}^{2}\end{array}\right]$. It is well-known that $A_{i}=n_{i} S_{i} \sim$ $W\left(n_{i}-1, \Sigma_{i}\right)$, i.e. it has a Wishart distribution with $n_{i}-1$ degrees of freedom and parameter $\Sigma_{i}$. Since $\Sigma_{i}$ is a positive definite matrix, there is a unique lower triangular matrix, $L_{i}$, such that $L_{i} L_{i}^{\prime}=\Sigma_{i}$ (Cholesky decomposition) and it is easily verified that

$$
P_{i}=L_{i}^{-1} A_{i} L_{i}^{\prime-1} \sim W\left(n_{i}-1, I\right),
$$

where $I$ is the identity matrix. Put $P_{i}=C_{i} C_{i}^{\prime}$. From Theorem 3.2.14 of Muirhead (1982), the elements of matrix $C_{i}=\left[\begin{array}{cc}V_{i} & 0 \\ N_{i} & W_{i}\end{array}\right]$ are independent and distributed as

$$
V_{i}^{2} \sim \chi_{\left(n_{i}-1\right)}^{2}, W_{i}^{2} \sim \chi_{\left(n_{i}-1\right)}^{2} \text { and } N_{i} \sim N(0,1) .
$$

It can be shown that matrix $L_{i}$ has the following form:

$$
L_{i}=\left[\begin{array}{cc}
\sigma_{1 i} & 0 \\
\rho_{i} \sigma_{2 i} & \sigma_{2 i} \sqrt{1-\rho_{i}^{2}}
\end{array}\right] .
$$


Therefore, we have

$$
\begin{aligned}
A_{i} & =\left(A_{k l}^{(i)}\right) \stackrel{d}{=} L_{i} C_{i} C_{i}^{\prime} L_{i}^{\prime} \\
& =\left[\begin{array}{cc}
\sigma_{1 i}^{2} V_{i}^{2} & \sigma_{1 i} \sigma_{2 i} \sqrt{1-\rho_{i}^{2}}\left(\tilde{\rho}_{i} V_{i}^{2}+N_{i} V_{i}\right) \\
\sigma_{1 i} \sigma_{2 i} \sqrt{1-\rho_{i}^{2}}\left(\tilde{\rho}_{i} V_{i}^{2}+N_{i} V_{i}\right) & \sigma_{2 i}^{2}\left(1-\rho_{i}^{2}\right)\left[\left(\tilde{\rho}_{i} V_{i}+N_{i}\right)^{2}+W_{i}^{2}\right]
\end{array}\right] .
\end{aligned}
$$

Since $R_{i}=\frac{A_{12}^{(i)}}{\sqrt{A_{11}^{(i)} A_{22}^{(i)}}}$, the proof is completed.

\section{Testing the Equality of Two Correlation Coeffi- cients}

In this section, we consider the problem of testing the equality of two independent correlation coefficients. At first, we propose the method of MSLR and give an algorithm that can be used for this problem. Then, we review two other existing approaches.

\subsection{Modified Signed Log-Likelihood Ratio Test}

It is easily verified that the SLR test statistic to test the hypothesis in (1) has the following form

$$
\begin{aligned}
\operatorname{SLR} & =\sqrt{2} \operatorname{sign}\left(r_{1}-r_{2}\right) \sqrt{2(\ell(\hat{\boldsymbol{\theta}})-\ell(\tilde{\boldsymbol{\theta}}))} \\
& =\operatorname{sign}\left(r_{1}-r_{2}\right)\left(\sum_{i=1}^{2} n_{i} \log \left(\frac{\left(1-\tilde{\rho} r_{i}\right)^{2}}{\left(1-r_{i}^{2}\right)\left(1-\tilde{\rho}^{2}\right)}\right)\right)^{\frac{1}{2}}
\end{aligned}
$$

where $\hat{\boldsymbol{\theta}}=\left(\hat{\boldsymbol{\theta}}_{1}, \hat{\boldsymbol{\theta}}_{2}\right)$ and $\tilde{\boldsymbol{\theta}}=\left(\tilde{\boldsymbol{\theta}}_{1}, \tilde{\boldsymbol{\theta}}_{2}\right)$, and $\operatorname{sign}(x)=1$, if $x>0$ and $\operatorname{sign}(x)=$ -1 , if $x<0$.

It is well known that SLR is asymptotically distributed as a standard normal distribution (Cox and Hinkley, 1979), and a p-value for testing the hypothesis in (1) is

$$
p=2\left(1-\Phi\left(\left|\mathrm{SL} R_{0}\right|\right)\right),
$$

where $\mathrm{SLR}_{0}$ is the observed value of the statistic SLR and $\Phi(t)$ is the standard normal distribution function. 
If we use the estimator $R_{F}$ instead of $\tilde{\rho}$, the SLR statistic in (6) is rewritten as

$$
\mathrm{SLR}=\operatorname{sign}\left(r_{1}-r_{2}\right)\left(\sum_{i=1}^{k} n_{i} \log \left\{\frac{\left(1-R_{F} r_{i}\right)^{2}}{\left(1-r_{i}^{2}\right)\left(1-R_{F}^{2}\right)}\right\}\right)^{\frac{1}{2}}
$$

Pierce and Peters (1992) showed the SLR test is not very accurate, and some modifications are needed to increase the accuracy of the SLR. There exist various ways to improve the accuracy of this approximation by adjusting the SLR statistic. For the various ways to improve the accuracy of SLR method, refer to the works of Barndorff-Nielsen (1986, 1991), Skovgaard (2001), and DiCiccio et al. (2001). We used the method proposed by DiCiccio et al. (2001), which has the following form

$$
\mathrm{MSLR}=\frac{\mathrm{SLR}-m(\mathrm{SLR})}{\sqrt{v(\mathrm{SLR})}},
$$

where $m$ (SLR) and $v(\mathrm{SLR})$ are the mean and variance of the SLR statistic evaluated at the constrained MLE's of the model parameters and is asymptotically distributed as a standard normal distribution.

Krishnamoorthy and Lee (2014) used the parametric bootstrap approach to approximate the mean and variance of the MSLR test statistic for the problem of testing the equality of normal coefficients of variation. We use this approach for the problem of testing the equality of two normal correlation coefficients. In Section 4, using Monte Carlo simulation, we will show that this new method is more accurate than the other competing methods. This approach is given in the following algorithm:

Algorithm 1. Given $r_{1}$ and $r_{2}$,

1. Compute $r_{F}$, the observed value of estimator $R_{F}$ in (4).

2. Generate $V_{i}^{2} \sim \chi_{\left(n_{i}-1\right)}^{2}, W_{i}^{2} \sim \chi_{\left(n_{i}-2\right)}^{2}, N_{i} \sim N(0,1), i=1,2$.

3. Compute $r_{F}^{*}=r_{F} / \sqrt{1-r_{F}^{2}}$.

4. Compute $r_{i}^{*}$ by substituting $r_{F}$ instead of $\rho$ in (5) as

$$
r_{i}^{*}=\frac{r_{F}^{*} V_{i}+N_{i}}{\sqrt{\left(r_{F}^{*} V_{i}+N_{i}\right)^{2}+W_{i}^{2}}} .
$$

J. Statist. Res. Iran 12 (2015): 147-162 
5. Compute the test statistic SLR in (8).

6. Repeat steps 3-5 for a large number of times (say $\mathrm{M}=10,000$ ).

7. Compute the sample mean and sample variance of SLR and compute the MSLR in (9).

8. Determine the p-value for testing $H_{0}: \rho_{1}=\rho_{2}$ vs $H_{1}: \rho_{1} \neq \rho_{2}$ as

$$
\mathrm{p}-\text { value }=2(1-\Phi(|\mathrm{MSLR}|)) .
$$

\subsection{Fisher's Z-transform}

It is well-known that

$$
Z_{i}=\frac{1}{2} \log \left(\frac{1+R_{i}}{1-R_{i}}\right)=\tanh ^{-1}\left(R_{i}\right),
$$

has asymptotic normal distribution with mean $\tanh ^{-1}\left(\rho_{i}\right)$ and variance $\frac{1}{n_{i}-3}$. Therefore, a test statistic for testing $H_{0}: \rho_{1}=\rho_{2}$, vs $H_{1}: \rho_{1} \neq \rho_{2}$ can be given by extending the one-sample Fisher's Z-transformation to the twosample case. Consider the following test statistic

$$
F Z=\frac{Z_{1}-Z_{2}}{\sqrt{\frac{1}{n_{1}-3}+\frac{1}{n_{2}-3}}} .
$$

Then, $F Z$ has asymptotic standard normal distribution, and the null hypothesis is rejected if $|F Z|>z_{\alpha / 2}$. For more details, refer to Zar (1999) and Krishnamoorthy and Xia (2007).

\subsection{Generalized Test Variable}

Krishnamoorthy and Xia (2007) proposed a generalized pivotal variable for $\rho_{i}$ as

$$
G_{\rho_{i}}=\frac{r_{i}^{*} W_{i}-Z_{i}}{\sqrt{\left(r_{i}^{*} W_{i}-Z_{i}\right)^{2}+V_{i}^{2}}}, \quad i=1,2,
$$

where $r_{i}^{*}=\frac{r_{i}}{\sqrt{1-r_{i}^{2}}}$, and $V_{i}, W_{i}$, and $Z_{i}$ are independent random variables with

$$
V_{i}^{2} \sim \chi_{\left(n_{i}-1\right)}^{2}, \quad W_{i}^{2} \sim \chi_{\left(n_{i}-2\right)}^{2}, \quad Z_{i} \sim N(0,1) .
$$


Therefore, a generalized pivotal variable for $\rho_{1}-\rho_{2}$ is given as

$$
G_{\rho}=G_{\rho_{1}}-G_{\rho_{2}} .
$$

So, the generalized p-value for testing $H_{0}: \rho_{1}=\rho_{2}$ vs $H_{1}: \rho_{1} \neq \rho_{2}$ is given by

$$
p=2 \min \left\{P\left(G_{\rho}<0\right), P\left(G_{\rho}>0\right)\right\} .
$$

\section{Numerical Study}

\subsection{Simulation Study}

We performed a simulation study with 10,000 replications to evaluate and compare the actual sizes of three approaches: the modified signed likelihood ratio test (MSLR), Fisher's Z-transform (FZ) test, and generalized test variable $(\mathrm{GV})$. We generate random samples of size $n_{1}$ and $n_{2}$ from two independent bivariate normal distributions for different values of common correlation $\rho=0.0,0.1,0.2, \ldots, 0.9$. We obtained the sample correlation coefficient and then the $p$-values of the MSLR, FZ and GV to test the hypothesis $H_{0}: \rho_{1}=\rho_{2}$ vs $H_{1}: \rho_{1} \neq \rho_{2}$. Here, we consider the nominal level $\alpha=0.05$. The results are given in Table 1 .

We can conclude that

i. The actual size of MSLR test is satisfactory for all different values of common correlation coefficient and sample sizes.

ii. The actual size of FZ test is smaller than the nominal level when the sample sizes are small,

iii. The actual size of GV test is very smaller than the nominal level when $n_{1}$ is small and $n_{2}$ is large.

Since, the MSLR test is the only test that controls the correct frequency of rejected hypotheses in all cases, we recommend the MSLR for practical applications.

We also performed a simulation study to compare the powers of the considered approaches. The results are given in Tables 2 and 3 . It can be concluded that the powers of the three tests MSLR, GV and Fisher Ztransform are close when the sample sizes are equal. But the power of MSLR is larger than powers of GV and Fisher Z-transform when the sample sizes are unequal. 
Table 1. The actual sizes of the tests at nominal level $\alpha=0.05$.

\begin{tabular}{|c|c|c|c|c|c|c|}
\hline \multirow[b]{2}{*}{$\rho$} & \multirow[b]{2}{*}{ Method } & \multicolumn{5}{|c|}{$n_{1}, n_{2}$} \\
\hline & & 5,5 & 5,10 & 10,10 & 5,15 & 5,25 \\
\hline \multirow[t]{3}{*}{0} & MSLR & 0.052 & 0.050 & 0.051 & 0.056 & 0.049 \\
\hline & $\mathrm{FZ}$ & 0.046 & 0.049 & 0.048 & 0.045 & 0.052 \\
\hline & $\mathrm{GV}$ & 0.052 & 0.049 & 0.053 & 0.047 & 0.040 \\
\hline \multirow[t]{3}{*}{0.1} & MSLR & 0.053 & 0.050 & 0.055 & 0.050 & 0.053 \\
\hline & FZ & 0.043 & 0.047 & 0.050 & 0.046 & 0.047 \\
\hline & $\mathrm{GV}$ & 0.051 & 0.048 & 0.054 & 0.044 & 0.036 \\
\hline \multirow[t]{3}{*}{0.2} & MSLR & 0.052 & 0.051 & 0.049 & 0.051 & 0.048 \\
\hline & $\mathrm{FZ}$ & 0.045 & 0.045 & 0.050 & 0.051 & 0.046 \\
\hline & $\mathrm{GV}$ & 0.052 & 0.050 & 0.051 & 0.047 & 0.038 \\
\hline \multirow[t]{3}{*}{0.3} & MSLR & 0.051 & 0.048 & 0.050 & 0.050 & 0.048 \\
\hline & $\mathrm{FZ}$ & 0.043 & 0.045 & 0.048 & 0.046 & 0.050 \\
\hline & $\mathrm{GV}$ & 0.051 & 0.051 & 0.051 & 0.045 & 0.033 \\
\hline \multirow[t]{3}{*}{0.4} & MSLR & 0.053 & 0.049 & 0.048 & 0.050 & 0.051 \\
\hline & $\mathrm{FZ}$ & 0.041 & 0.043 & 0.049 & 0.047 & 0.045 \\
\hline & $\mathrm{GV}$ & 0.050 & 0.051 & 0.052 & 0.044 & 0.036 \\
\hline \multirow[t]{3}{*}{0.5} & MSLR & 0.051 & 0.049 & 0.049 & 0.050 & 0.048 \\
\hline & $\mathrm{FZ}$ & 0.047 & 0.045 & 0.049 & 0.044 & 0.045 \\
\hline & $\mathrm{GV}$ & 0.051 & 0.049 & 0.051 & 0.047 & 0.037 \\
\hline \multirow[t]{3}{*}{0.6} & MSLR & 0.054 & 0.050 & 0.048 & 0.048 & 0.049 \\
\hline & $\mathrm{FZ}$ & 0.045 & 0.046 & 0.049 & 0.042 & 0.045 \\
\hline & $\mathrm{GV}$ & 0.051 & 0.049 & 0.051 & 0.048 & 0.035 \\
\hline \multirow[t]{3}{*}{0.7} & MSLR & 0.049 & 0.052 & 0.051 & 0.050 & 0.050 \\
\hline & $\mathrm{FZ}$ & 0.040 & 0.046 & 0.052 & 0.044 & 0.045 \\
\hline & $\mathrm{GV}$ & 0.053 & 0.050 & 0.051 & 0.045 & 0.036 \\
\hline \multirow[t]{3}{*}{0.8} & MSLR & 0.054 & 0.049 & 0.049 & 0.050 & 0.052 \\
\hline & $\mathrm{FZ}$ & 0.038 & 0.045 & 0.049 & 0.046 & 0.044 \\
\hline & $\mathrm{GV}$ & 0.051 & 0.051 & 0.051 & 0.047 & 0.037 \\
\hline \multirow[t]{3}{*}{0.9} & MSLR & 0.053 & 0.055 & 0.051 & 0.052 & 0.049 \\
\hline & $\mathrm{FZ}$ & 0.041 & 0.044 & 0.045 & 0.044 & 0.046 \\
\hline & GV & 0.051 & 0.048 & 0.053 & 0.045 & 0.039 \\
\hline
\end{tabular}


Table 2. Empirical powers of the tests at nominal level $\alpha=0.05$ with $\rho_{1}=0.05$.

\begin{tabular}{|c|c|c|c|c|c|c|c|c|c|}
\hline \multirow[b]{2}{*}{$\rho$} & \multirow[b]{2}{*}{ Method } & \multicolumn{8}{|c|}{$n_{1}, n_{2}$} \\
\hline & & 5,5 & 5,10 & 10,10 & 15,10 & 5,15 & 5,25 & 20,20 & 25,25 \\
\hline \multirow[t]{3}{*}{0.15} & MSLR & 0.052 & 0.055 & 0.057 & 0.063 & 0.061 & 0.069 & 0.073 & 0.078 \\
\hline & $\mathrm{FZ}$ & 0.049 & 0.046 & 0.051 & 0.048 & 0.046 & 0.052 & 0.074 & 0.080 \\
\hline & GV & 0.058 & 0.056 & 0.057 & 0.055 & 0.044 & 0.043 & 0.077 & 0.079 \\
\hline \multirow[t]{3}{*}{0.25} & MSLR & 0.059 & 0.064 & 0.067 & 0.082 & 0.063 & 0.076 & 0.111 & 0.123 \\
\hline & $\mathrm{FZ}$ & 0.051 & 0.056 & 0.068 & 0.072 & 0.052 & 0.059 & 0.105 & 0.135 \\
\hline & GV & 0.061 & 0.057 & 0.066 & 0.070 & 0.048 & 0.042 & 0.112 & 0.133 \\
\hline \multirow[t]{3}{*}{0.35} & MSLR & 0.069 & 0.071 & 0.088 & 0.111 & 0.088 & 0.089 & 0.172 & 0.201 \\
\hline & $\mathrm{FZ}$ & 0.056 & 0.064 & 0.091 & 0.094 & 0.067 & 0.067 & 0.178 & 0.214 \\
\hline & GV & 0.070 & 0.063 & 0.094 & 0.095 & 0.057 & 0.054 & 0.177 & 0.212 \\
\hline \multirow[t]{3}{*}{0.45} & MSLR & 0.076 & 0.085 & 0.097 & 0.164 & 0.099 & 0.108 & 0.251 & 0.324 \\
\hline & $\mathrm{FZ}$ & 0.066 & 0.067 & 0.117 & 0.133 & 0.080 & 0.084 & 0.252 & 0.313 \\
\hline & GV & 0.080 & 0.074 & 0.122 & 0.135 & 0.070 & 0.064 & 0.255 & 0.317 \\
\hline \multirow[t]{3}{*}{0.55} & MSLR & 0.087 & 0.105 & 0.151 & 0.226 & 0.115 & 0.123 & 0.377 & 0.442 \\
\hline & $\mathrm{FZ}$ & 0.071 & 0.082 & 0.153 & 0.181 & 0.100 & 0.102 & 0.351 & 0.422 \\
\hline & GV & 0.091 & 0.085 & 0.153 & 0.185 & 0.079 & 0.073 & 0.355 & 0.425 \\
\hline \multirow[t]{3}{*}{0.65} & MSLR & 0.102 & 0.131 & 0.201 & 0.290 & 0.136 & 0.171 & 0.456 & 0.549 \\
\hline & $\mathrm{FZ}$ & 0.092 & 0.101 & 0.208 & 0.235 & 0.119 & 0.122 & 0.448 & 0.541 \\
\hline & GV & 0.121 & 0.108 & 0.204 & 0.236 & 0.099 & 0.091 & 0.435 & 0.538 \\
\hline \multirow[t]{3}{*}{0.75} & MSLR & 0.113 & 0.151 & 0.245 & 0.372 & 0.192 & 0.189 & 0.528 & 0.638 \\
\hline & $\mathrm{FZ}$ & 0.098 & 0.124 & 0.240 & 0.291 & 0.139 & 0.140 & 0.540 & 0.650 \\
\hline & GV & 0.125 & 0.121 & 0.252 & 0.291 & 0.123 & 0.114 & 0.546 & 0.651 \\
\hline \multirow[t]{3}{*}{0.85} & MSLR & 0.131 & 0.175 & 0.290 & 0.448 & 0.214 & 0.235 & 0.652 & 0.776 \\
\hline & $\mathrm{FZ}$ & 0.119 & 0.142 & 0.297 & 0.348 & 0.167 & 0.162 & 0.638 & 0.741 \\
\hline & GV & 0.134 & 0.139 & 0.294 & 0.363 & 0.152 & 0.133 & 0.643 & 0.739 \\
\hline \multirow[t]{3}{*}{0.95} & MSLR & 0.143 & 0.208 & 0.356 & 0.523 & 0.253 & 0.264 & 0.709 & 0.823 \\
\hline & $\mathrm{FZ}$ & 0.129 & 0.168 & 0.349 & 0.414 & 0.193 & 0.196 & 0.717 & 0.814 \\
\hline & $\mathrm{GV}$ & 0.155 & 0.171 & 0.355 & 0.410 & 0.173 & 0.158 & 0.710 & 0.813 \\
\hline
\end{tabular}


Table 3. Empirical powers of the tests at nominal level $\alpha=0.05$ with $\rho_{1}=0.05$.

\begin{tabular}{|c|c|c|c|c|c|c|c|c|c|}
\hline \multirow[b]{2}{*}{$\rho$} & \multirow[b]{2}{*}{ Method } & \multicolumn{8}{|c|}{$n_{1}, n_{2}$} \\
\hline & & 5,5 & 5,10 & 10,10 & 15,10 & 5,15 & 5,25 & 20,20 & 25,25 \\
\hline \multirow[t]{3}{*}{-0.15} & MSLR & 0.054 & 0.058 & 0.066 & 0.072 & 0.058 & 0.061 & 0.063 & 0.064 \\
\hline & $\mathrm{FZ}$ & 0.049 & 0.051 & 0.068 & 0.069 & 0.053 & 0.053 & 0.075 & 0.077 \\
\hline & $\mathrm{GV}$ & 0.063 & 0.056 & 0.071 & 0.071 & 0.048 & 0.038 & 0.071 & 0.079 \\
\hline \multirow[t]{3}{*}{-0.25} & MSLR & 0.066 & 0.072 & 0.084 & 0.113 & 0.064 & 0.071 & 0.103 & 0.145 \\
\hline & FZ & 0.058 & 0.068 & 0.090 & 0.099 & 0.058 & 0.057 & 0.119 & 0.137 \\
\hline & GV & 0.071 & 0.061 & 0.089 & 0.100 & 0.055 & 0.044 & 0.114 & 0.133 \\
\hline \multirow[t]{3}{*}{-0.35} & MSLR & 0.074 & 0.089 & 0.116 & 0.121 & 0.080 & 0.083 & 0.183 & 0.207 \\
\hline & $\mathrm{FZ}$ & 0.060 & 0.074 & 0.117 & 0.121 & 0.068 & 0.068 & 0.179 & 0.211 \\
\hline & GV & 0.080 & 0.074 & 0.120 & 0.141 & 0.061 & 0.055 & 0.176 & 0.212 \\
\hline \multirow[t]{3}{*}{-0.45} & MSLR & 0.085 & 0.114 & 0.164 & 0.192 & 0.101 & 0.092 & 0.246 & 0.319 \\
\hline & FZ & 0.075 & 0.092 & 0.162 & 0.188 & 0.078 & 0.078 & 0.249 & 0.319 \\
\hline & $\mathrm{GV}$ & 0.090 & 0.092 & 0.164 & 0.186 & 0.077 & 0.065 & 0.257 & 0.314 \\
\hline \multirow[t]{3}{*}{-0.55} & MSLR & 0.106 & 0.133 & 0.205 & 0.247 & 0.116 & 0.118 & 0.366 & 0.418 \\
\hline & $\mathrm{FZ}$ & 0.090 & 0.108 & 0.206 & 0.247 & 0.101 & 0.101 & 0.349 & 0.426 \\
\hline & $\mathrm{GV}$ & 0.109 & 0.107 & 0.208 & 0.252 & 0.086 & 0.081 & 0.346 & 0.432 \\
\hline \multirow[t]{3}{*}{-0.65} & MSLR & 0.121 & 0.166 & 0.252 & 0.278 & 0.136 & 0.158 & 0.417 & 0.569 \\
\hline & $\mathrm{FZ}$ & 0.105 & 0.130 & 0.248 & 0.304 & 0.122 & 0.122 & 0.439 & 0.541 \\
\hline & $\mathrm{GV}$ & 0.119 & 0.126 & 0.257 & 0.299 & 0.100 & 0.098 & 0.448 & 0.542 \\
\hline \multirow[t]{3}{*}{-0.75} & MSLR & 0.144 & 0.194 & 0.308 & 0.385 & 0.179 & 0.196 & 0.565 & 0.620 \\
\hline & $\mathrm{FZ}$ & 0.120 & 0.154 & 0.304 & 0.372 & 0.143 & 0.143 & 0.546 & 0.651 \\
\hline & GV & 0.143 & 0.150 & 0.312 & 0.368 & 0.127 & 0.113 & 0.552 & 0.651 \\
\hline \multirow[t]{3}{*}{-0.85} & MSLR & 0.161 & 0.216 & 0.368 & 0.445 & 0.188 & 0.240 & 0.660 & 0.734 \\
\hline & $\mathrm{FZ}$ & 0.142 & 0.174 & 0.364 & 0.440 & 0.166 & 0.166 & 0.630 & 0.750 \\
\hline & GV & 0.166 & 0.181 & 0.368 & 0.442 & 0.147 & 0.135 & 0.638 & 0.735 \\
\hline \multirow[t]{3}{*}{-0.95} & MSLR & 0.179 & 0.253 & 0.430 & 0.505 & 0.254 & 0.268 & 0.709 & 0.819 \\
\hline & FZ & 0.158 & 0.202 & 0.425 & 0.511 & 0.193 & 0.193 & 0.716 & 0.813 \\
\hline & GV & 0.192 & 0.199 & 0.424 & 0.512 & 0.176 & 0.164 & 0.717 & 0.821 \\
\hline
\end{tabular}


Table 4. correlations between laterality of blood flow in three brain regions and verbal memory score.

\begin{tabular}{cccc}
\hline & \multicolumn{3}{c}{ laterality of blood flow } \\
\cline { 2 - 4 } Gender & Temporal & Subcortical & Frontal \\
\hline Male & $r_{\mathrm{M}, \mathrm{vt}}=-0.340$ & $r_{\mathrm{M}, \mathrm{vs}}=0.641$ & $r_{\mathrm{M}, \mathrm{vf}}=-0.032$ \\
Female & $r_{\mathrm{F}, \mathrm{vt}}=0.812$ & $r_{\mathrm{F}, \mathrm{vs}}=0.491$ & $r_{\mathrm{F}, \mathrm{vf}}=-0.212$ \\
\hline
\end{tabular}

Table 5. P-values of the tests for equality of correlations between laterality of blood flow in three brain regions and verbal memory score.

\begin{tabular}{lccc}
\hline \hline method & $\rho_{\mathrm{M}, \mathrm{vt}}=\rho_{\mathrm{F}, \mathrm{vt}}$ & $\rho_{\mathrm{M}, \mathrm{vs}}=\rho_{\mathrm{F}, \mathrm{vs}}$ & $\rho_{\mathrm{M}, \mathrm{vf}}=\rho_{\mathrm{F}, \mathrm{vf}}$ \\
\hline MSLR & 0.0008 & 0.5978 & 0.6677 \\
GV & 0.0008 & 0.5948 & 0.6682 \\
Fisher Z & 0.0004 & 0.6018 & 0.6673 \\
\hline
\end{tabular}

\subsection{Real Data}

In this example, we test the equality of two independent correlations in three groups of data. This data set is given by Bilker et al. (2004) and also have been analyzed by Krishnamoorthy and Xia (2007).

For each of two groups of 14 men and 14 women, the sample correlation between a verbal memory score $(\mathrm{v})$ and laterality of blood flow in each of three brain regions, namely, temporal (t), frontal (f) and subcortical (s) are obtained in Table 4. It may be of interest to compare the correlations between the laterality of blood flow in each brain region and verbal memory score across gender. The results are given in Table 5 . We can find that there is no significant difference between male and female correlations in frontal and subcortical cases.

\section{Conclusion}

Existing methods for comparing the correlation coefficients of two independent bivariate normal distributions do not perform well in a range of smallsample settings. Krishnamoorthy and Xia (2007) obtained a generalized pivotal quantity for difference of two correlation coefficients and they gave a method for testing the equality of two correlation coefficients using this generalized pivotal quantity. By using a simulation study, they showed the 
test size of their method is greater than the nominal level for small sample sizes. In other words, they showed that their method is liberal.

Using the method of modified signed log likelihood, Krishnamoorthy and Lee (2014) considered the problem of equality of coefficients of variation for independent normal populations. This method is an exact method to test a hypothesis for unknown parameter. The accuracy of this method is very satisfactory such that the actual size of test is approximately close to nominal level even for small sample sizes. In this article, we used the MSLR method for testing the equality of two independent correlation coefficients because of the accuracy of this method for inference about the unknown model parameter and that the other competing methods have deviations that we cannot rely to them. In this paper, we explained the generating the sample correlation coefficients of a bivariate normal distribution (See Lemma 2.1). Then, we obtained the MSLR test statistics for the problem of the equality of two correlation coefficients. All the mathematical formulas obtained in our paper are different from that of used in chapter 4 of Krishnamoorthy and Xia (2007). As we see, for using the MSLR method, one should consider the MLEs of the unknown parameters but the MLE was not stated in Krishnamoorthy and Xia (2007) at all. We compared our method with the method of GV used by Krishnamoorthy and Xia (2007) and another competing method by simulation studies. We found that MSLR test always are satisfactory in terms of actual sizes regardless of the sample sizes and values of the common correlation coefficient in comparison with existing methods. Also, the power of MSLR is larger than powers of GV and Fisher Z-transform when the sample sizes are unequal.

\section{Acknowledgement}

The authors are grateful to the Editor-in-Chief and referees for their helpful comments and suggestions on improving the initial version of this manuscript.

\section{References}

Barndorff-Nielsen, O.E. (1986). Inference on Full or Partial Parameters based on the Standardized Signed Log Likelihood Ratio. Biometrika, 73, 307-322.

Barndorff-Nielsen, O.E. (1991). Modified Signed Log Likelihood Ratio. Biometrika, 78, 557563. 
Bilker, W.B., Brensinger, C. and Gur, R.C. (2004). A Two Factor ANOVA-like Test for Correlated Correlations: CORANOVA. Multivariate Behavioral Research, 39, 565-594.

Cox, D.R. and Hinkley, D.V. (1979). Theoretical Statistics. Chapman and Hall, London.

DiCiccio, T.J., Martin, M.A. and Stern, S.E. (2001). Simple and Accurate One-sided Inference from Signed Roots of Likelihood Ratios. Canadian Journal of Statistics, 29, 67-76.

Donner, A. and Rosner, B. (1980). On Inferences Concerning a Common Correlation Coefficient. Applied Statistics, 29, 69-76.

Fisher, R.A. (1915). Frequency Distribution of the Values of the Correlation Coefficient in Samples from an Indefinitely Large Population. Biometrika, 10, 507-521.

Fisher, R.A. (1921). On the "Probable Error" of a Coefficient of Correlation Deduced from a Small Sample. Metron, 1, 3-32.

Hotelling, H. (1953). New Light on the Correlation Coefficient and Its Transforms. Journal of the Royal Statistical Society. Series B (Methodological), 15, 193-232.

Kazemi, M.R. and Jafari, A.A. (2015). Comparing Seventeen Interval Estimates for a Bivariate Normal Correlation Coefficient. Journal of Statistics Applications 8 Probability Letters, 2, 23-35.

Krishnamoorthy, K. and Lee, M. (2014). Improved Tests for the Equality of Normal Coefficients of Variation. Computational Statistics, 29, 215-232.

Krishnamoorthy, K. and Xia, Y. (2007). Inferences on Correlation Coefficients: One-sample, Independent and Correlated Cases. Journal of Statistical Planning and Inference, 137, 23622379 .

Muirhead, R.J. (1982). Aspects of Multivariate Statistical Theory. Wiley, New York.

Olkin, I. and Finn, J.D. (1995). Correlations Redux. Psychological Bulletin, 118, 155-164.

Pearson, K. (1933). On a Method of Determining whether a Sample of Size n Supposed to have been Drawn from a Parent Population Having a Known Probability Integral has Probably been Drawn at Random. Biometrika, 25, 379-410.

Pierce, D.A. and Peters, D. (1992). Practical Use of Higher Order Asymptotics for Multiparameter Exponential Families. Journal of the Royal Statistical Society. Series B (Methodological), 54, 701-737.

Skovgaard, I.M. (2001). Likelihood Asymptotics. Scandinavian Journal of Statistics, 28, $3-32$.

Sun, Y. and Wong, A. (2007). Interval Estimation for the Normal Correlation Coefficient. Statistics and Probability Letters, 77, 1652-1661. 
Zar, J.H. (1999). Biostatistical Analysis. Prentice Hall, India, 4th edition.

\section{Mohammad Reza Kazemi}

Department of Statistics,

Fasa University,

Fasa, Iran

email:kazemi@fasau.ac.ir

\section{Ali Akbar Jafari}

Department of Statistics,

Yazd University,

Yazd, Iran.

email: aajafari@yazd.ac.ir 